\title{
Attitudes envers la langue française pendant les années 1840
}

\section{Stephen Kenny}

Volume 34, numéro 2, septembre 1980

URI : https://id.erudit.org/iderudit/303859ar

DOI : https://doi.org/10.7202/303859ar

Aller au sommaire du numéro

Éditeur(s)

Institut d'histoire de l'Amérique française

ISSN

0035-2357 (imprimé)

1492-1383 (numérique)

Découvrir la revue

\section{Citer cette note}

Kenny, S. (1980). Attitudes envers la langue française pendant les années 1840. Revue d'histoire de l'Amérique française, 34(2), 257-267.

https://doi.org/10.7202/303859ar d'utilisation que vous pouvez consulter en ligne.

https://apropos.erudit.org/fr/usagers/politique-dutilisation/ 


\title{
NOTES DE RECHERCHE
}

\author{
I \\ ATTITUDES ENVERS LA LANGUE FRANÇAISE \\ PENDANT LES ANNÉS 1840*
}

STEPHEN KENNY

Département d'histoire

Université du Vermont

En 1841, les Canadiens entraient dans une nouvelle communauté politique qui leur était imposée. L'intention du gouvernement britannique était de faire du Canada une colonie anglaise. Acceptable pour bon nombre de Canadiens, ce but était par contre inadmissible pour ceux qui parlaient français. L'objet de la présente note est d'examiner quelques-unes des opinions exprimées et des attitudes prises au sujet de la langue française au cours de cette période difficile de l'histoire des Canadiens français. Nous espérons montrer l'importance des efforts fournis pour préserver le français au Canada et souligner la force de la communauté francophone qui, à cette époque, refusa de céder aux pressions considérables qui la menaçaient.

L'article 41 du nouvel Acte d'Union faisait de l'anglais la seule langue officielle de la province du Canada ${ }^{1}$. Bien que le français fût toujours toléré dans les débats de l'Assemblée, le désir du gouvernement britannique d'assimiler les Canadiens français était catégori$q^{2}{ }^{2}$. D'ailleurs, la politique linguistique ne fut pas le seul exemple de cette politique. Partout dans la colonie, les Canadiens, français et anglais, percevaient bien les buts de l'Union. Par exemple, John Neilson, rédacteur en chef du Quebec Gazette, s'opposait à la nouvelle Union, prétendant qu'elle était injuste et humiliante et que seu-

\footnotetext{
* Je tiens à remercier Jacques Pâquet, membre du département d'Éducation à l'université de Calgary, qui m'a aidé à la rédaction de cette note de recherche.

1 Documents of the Canadian Constitution, 1759-1915 (Toronto, 1918), selected and edited by W. P. M. Kennedy. The Union Act, 1841 (3 et 4 Victoria, cap. 35).

2 Voir: Lord Durham's Report Toronto, 1963), edited with an introduction by Gerald M. Craig, 22-23, 148-149. Voir aussi: RG7 G12 Vol. 54. Sydenham à Russel (confidentiel) (Montréal, 9 mars 1840).
}

RHAF, vol. 34, no 2, septembre 1980 
le son absurdité dépassait son injustice. Il la considérait comme une tentative malhonnête de changer " les lois, la langue, les us et coutumes " de la moitié de la population canadienne ${ }^{3}$. Mais tous les journalistes canadiens-anglais ne faisaient pas les mêmes objections. L'auteur d'un article paru en juin 1841 dans le Toronto Monthly Review, un magazine éphémère de cette époque, affirmait que la nouvelle union politique signifiait la mort de la langue française au Canada; il se demandait pourquoi les Canadiens français s'obstinaient à vouloir préserver une langue qui en faisait des étrangers dans leur propre pays et qui assurait leur infériorité vis-à-vis de leurs voisins anglophones. Pour lui, la place privilégiée accordée à l'anglais était l'aspect le plus positif de l'Union et constituerait certainement un grand avantage pour les Canadiens français ${ }^{4}$.

C'est dans un article du Toronto Patriot, paru quelques mois après l'union des Canadas, que nous trouvons une des illustrations les plus frappantes de l'attitude des Canadiens anglais. Pour ce journal, la civilisation s'identifiait à la langue anglaise, destinée à prévaloir puisqu'elle était la langue des forces économiques et commerciales. En ce qui concerne l'avenir du français au Canada, le journal torontois s'exprimait avec des mots précis et provocants:

The sonorous tongue of the Spaniard of Florida has been lost in the busy hum of the active American: the voice of the French colonist has yielded in Louisiana, to the same progressing «dialect "; and the patois of the Lower Canadian habitant will as certainly become as rare and useless in legislative proceedings, as the half-forgotten gutturals of the Huron and thePottawatamie. ${ }^{5}$

De son côté la population francophone du Bas-Canada se rendait parfaitement compte que sa langue était sérieusement menacée. Peu de temps après la proclamation officielle du régime de 1841 , une lettre de François-Xavier Garneau au journal Le Canadien, déclarait qu'avec l'union des deux Canadas, le programme politique d'abolition de la langue française était sérieusement commencé ${ }^{6}$. Commentant la situation des Canadiens français, Napoléon Aubin, ré-

3 Quebec Gazette, mercredi, 26 août 1840.

4 Toronto Monthly Review, I, 1 (juin 1841). Pour d'autres exemples des opinions pareilles, voir: Literary Garland, I,3 (février 1839) et Major John Richardson, Eight Years in Canada, embracing a Review of the Administration of Lords Durham and Sydenham, Sir Charles Bagot and Lord Metcalfe and including numerous interesting letters from Ltd. Durham, Chas. Buller and other well known public characters (Montreal, 1847), 102.

5 Toronto Patriot, mardi, 27 avril 1841.

6 Le Canadien, lundi, 22 février 1841. 
dacteur du journal satirique Le Fantasque, écrivait cette phrase un peu facétieuse mais vraie:

Nous aimons nos chers frères d'en haut et ils nous aiment tant qu'ils voudraient nous manger. ${ }^{?}$

Peut-être un peu bizarre comme attitude, les Canadiens français, pour éviter d'être absorbés, se sont rendus compte qu'ils devaient apprendre à connaître la langue et le mode de vie des Anglais. En fait, de nombreux efforts furent faits pour répandre l'anglais. Par exemple, en 1839, Étienne Parent, rédacteur du journal $\mathrm{Le} C \mathrm{Ca}$ nadien, félicitait le Séminaire de Québec pour les progrès réalisés dans l'enseignement de cette langue. Il croyait que la connaissance de l'anglais était indispensable à ses compatriotes s'ils voulaient se protéger et maintenir leur culture ${ }^{8}$. Pendant les années 1840, plusieurs collèges introduisirent des cours d'anglais. Étienne Parent et d'autres encourageaient leurs efforts. Dans certains collèges, des sections complètes d'anglais furent ouvertes. Ainsi, le Collège de Sainte-Anne-de-la-Pocatière annonçait en 1840 que son programme prendrait une orientation plus pratique ${ }^{9}$ et que serait introduit l'enseignement de l'anglais et des techniques industrielles ${ }^{10}$. Même le gouverneur général, Lord Sydenham, envoyé au Canada pour y introduire l'Union, fit une donation de $£ 50$ à la nouvelle section anglaise $^{11}$. La construction commença en juin $1841^{12}$ et les nouveaux cours débutèrent à l'automne de $1842^{13}$. D'autres institutions, tels le Collège de Chambly ${ }^{14}$, le Séminaire de Nicolet ${ }^{15}$ et le Collège de l'Assomption ${ }^{16}$, annoncèrent aussi l'introduction prochaine de nouveaux cours d'anglais. L'importance de l'enseignement en anglais est illustrée par les frais demandés au Séminaire de Nicolet: le cours en anglais coûtait $£ 20$ par session tandis que le cours classique était réduit à $£ 16^{17}$.

De tels changements étaient encouragés. À l'automne 1840, dans une lettre de presque trois colonnes et demie publiée dans la Gazette de Québec et signé "un Canadien ", l'auteur commentait les

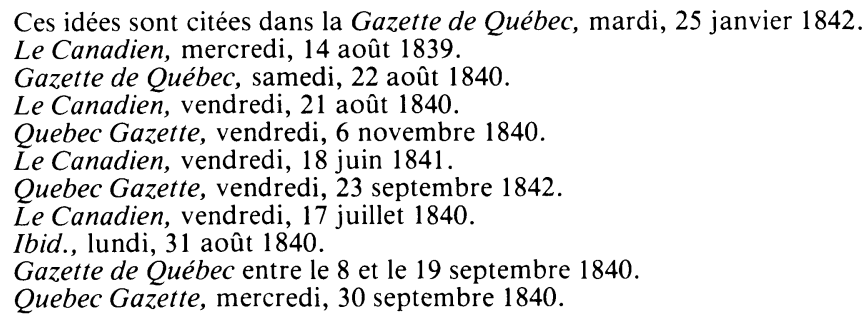


dangers auxquels les Canadiens français étaient exposés sous l'Union. Il se disait heureux de voir divers collèges introduire de nouveaux cours et il soulignait l'importance de l'avènement d'une éducation pratique dans le Bas-Canada. Il croyait que ses compatriotes se devaient de recevoir une éducation aussi pratique et aussi orientée vers l'industrie que les Anglais. Il les jugeait inférieurs à leurs compatriotes anglophones et souhaitait qu'ils se dotent d'un système d'éducation leur permettant de se hausser à leur niveau, autrement ils risqueraient de disparaître ${ }^{18}$. En fait, les opinions exprimées dans cette lettre étaient typiques de la pensée d'un bon nombre de ceux qui croyaient à la nécessité d'acquérir la langue anglaise.

L'attitude vis-à-vis de l'anglais, qu'on remarque chez plusieurs commentateurs canadiens-français, était réaliste et pragmatique. La facilité de s'exprimer en anglais était perçue comme un avantage dans la mesure où elle aidait la survivance de la nation canadiennefrançaise. Beaucoup de Canadiens français reconnaissaient que les habitants anglophones étaient doués d'un dynamisme et d'un sens du commerce qui leur faisaient défaut à eux. Apprendre l'anglais leur permettrait de devenir plus compétitifs et de jouer aussi un rôle dans le monde du commerce. Ils n'étaient nullement motivés par l'idée d'apprendre l'anglais pour la langue elle-même ni pour les valeurs culturelles qui s'y rattachaient ${ }^{19}$.

Nous trouvons un reflet de cette façon de penser dans une controverse entre La Minerve et la Montreal Gazette en 1843. Le journal français refusait d'admettre que les Canadiens français finiraient par être absorbés par les Canadiens anglais. Il admettait que les Anglais étaient plus actifs et de meilleurs hommes d'affaires, mais soutenait que cette supériorité n'était ni intrinsèque ni innée, mais qu'elle était due tout simplement au fait que les Anglais avaient de meilleurs rapports commerciaux avec la mère patrie ${ }^{20}$. Un autre exemple de cette attitude nous est fourni dans un discours sur l'enseignement primaire, prononcé par Amable Berthelot, député

18 Gazette de Québec, samedi, 10 octobre 1840.

19 Il est intéressant de noter que, dans son étude politique sur l'Union, Jacques Monet conclut que l'attachement des Canadiens français au système du gouvernement de l'Union était hautement réaliste et dicté par leurs intérêts. Leur loyauté était sans émotion et sans passion. Pour préserver leur nationalité, il fallait accepter le système. Voir: The Last Cannon Shot (Toronto, 1969), 399. À mon avis, il est un peu surprenant de découvrir que leur attitude à l'égard de la langue anglaise était aussi réaliste et pragmatique.

20 La Minerve, jeudi, 4 avril 1840. 
de Kamouraska à l'Assemblée législative et beau-père de LouisHippolyte LaFontaine. L'auteur y invoquait l'utilité de l'anglais pour en recommander l'enseignement:

J'aurais voulu démontrer que sous le point de vue des intérêts pécuniaires, les Anglais ont sur nous un avantage qui résulte de ce que leur langue est beaucoup plus facile que la nôtre, et aussi de ce que leur éducation tend plus vers le positif et l'utilité pratique que la nôtre. ${ }^{21}$

Étienne Parent reconnaissait aussi les qualités des Canadiens anglophones. Dans une série de conférences faites au cours de l'hiver 1846 à l'Institut Canadien, il souligna le danger de continuer à orienter la jeunesse canadienne-française vers les professions libérales et prétendit même que l'existence politique et nationale de la communauté s'en trouverait menacée et affaiblie ${ }^{22}$. Il croyait que l'ingéniosité et l'énergie des Anglais ne devaient pas leur être enviées mais qu'il fallait reconnaître ces qualités et les imiter ${ }^{23}$.

Peu de Canadiens français cependant allèrent aussi loin que Charles Mondelet, avocat et futur juge. Dans une série de lettres publiées en 1840, il proposait un système d'éducation qui pourrait supprimer les "odious national distinctions" et introduire au Canada une meilleure a mbiance ${ }^{24}$. Il croyait que des écoles des deux langues devaient être construites dans chaque localité et que les francophones iraient aux écoles anglaises tandis que les anglophones fréquenteraient les écoles françaises et que finalement, la méfiance et l'hostilité céderaient la place à une confiance mutuelle. Mais le projet de Mondelet avait aussi son aspect négatif, et cet avocat admettait lui-même qu'avec un tel système, l'anglais finirait par devenir la seule langue du Canada. Dès que tous pourraient parler la même langue, les distinctions nationales disparaîtraient. Sa conception de la destinée du français était fort vague, mais il espérait préserver cette langue d'une façon ou d'une autre ${ }^{25}$.

21 Amable Berthelot, Dissertation sur l'Instruction Primaire (Québec, imprimée par Augustin Côté et Cie, 1845), 9. Lue à la Société de Discussion de Québec, 12 mai 1845 .

22 Lecture Prononcée par Étienne Parent devant l'Institut Canadien, jeudi, 19 novembre 1841 (Montréal, l'Imprimerie de la Revue Canadienne, 1846), 4.

23 Ibid., 10.

24 Charles Mondelet, Lettres sur l'Éducation Élémentaire et Pratique (Montréal, imprimées par John James Williams, 1841), 9.

25 Ibid., 10. 
Il est donc clair que le désir d'apprendre l'anglais ne venait pas de l'amour de cette langue mais était dicté par la nécessité. Les Canadiens français devaient vaincre leur infériorité, et ils devaient devenir plus énergiques et plus dynamiques. Apprendre à parler l'anglais était un moyen d'y parvenir. En se mettant sur le même pied que les anglophones, ils seraient mieux armés pour défendre leur culture et leur nationalité. Tandis qu'ils comprenaient la nécessité d'apprendre l'anglais, ils n'étaient pas prêts à oublier le français et percevaient le danger que représentait l'expansion d'une langue étrangère au Bas-Canada. Par exemple, au fil des années, Le Fantasque nota avec répulsion un phénomène qu'il qualifia d'"anglomanie». Il se plaignit qu'à Québec tant d'enseignes de marchands et de magasins n'affichaient qu'en anglais et que l'aspect français de la ville se remarquait à peine. Il se moqua de la manière fort à la mode qu'avaient les dames canadiennes-françaises d'épicer leur conversation d'expressions anglaises telles que "Dear Me», "O Lord" et "Good Gracious». Le journal déplora aussi leur penchant pour les messieurs anglophones ${ }^{26}$.

Le pragmatisme des Canadiens français à l'égard de l'anglais ressort davantage si on le compare à leur attitude vis-à-vis de leur propre langue. Ils n'abandonnaient pas le français ni ne s'en désintéressaient mais, au contraire, ils le défendirent énergiquement au cours des années. En examinant leurs conceptions de la langue française, on voit que les Canadiens français ne la considéraient pas comme inadéquate ou inférieure aux autres langues. En fait, ils la croyaient supérieure et cette conception est illustrée par de multiples exemples.

Dans un éditorial paru au printemps de 1843, La Minerve affirmait que le français ne pouvait pas être comparé aux parlers d'Écosse et d'Irlande qui n'étaient que "des idiomes barbares", abandonnés à juste titre au profit d'une langue plus belle, l'anglais. Le journal irlandais, le Montreal Times, prit alors la défense du gaëlique, soutenant qu'il ne s'agissait pas d'un parler barbare et que l'article de La Minerve était insultant. Mais comment dès lors fallait-il concilier le français et l'anglais? La réponse était simple. Plutôt que de lutter vainement, les deux communautés, anglophone et francophone, devaient tirer profit de leurs richesses respectives ${ }^{27}$.

26 Le Fantasque, samedi, 18 novembre 1848.

27 La Minerve, jeudi, 27 avril et lundi, 1er mai 1843. 
Qu'il fût insultant ou non, peu nous importe ici; ce qui est important à nos yeux, c'est que de telles affirmations montrent bien que les Canadiens français ne considéraient pas leur langue inférieure à l'anglais et moins encore à l'écossais et à l'irlandais. La Revue Canadienne, une revue littéraire, nous fournit d'autres exemples de cette attitude. En 1845, son rédacteur en chef, Louis-Octave LeTourneux, écrivait dans le premier numéro que tous les Canadiens pouvaient vivre ensemble dans le bonheur et dans la paix sans se préoccuper d'hostilité et de rivalité nationales. Il suffisait que chaque groupe garde et chérisse sa langue et ses traditions tout en respectant celles des autres ${ }^{28}$. Cela était évidemment plus facile à dire qu'à faire. Des idées analogues étaient exprimées dans un discours au sujet de l'enseignement élémentaire que prononçait Augustin-Norbert Morin, député de Bellechasse à l'Assemblée législative et futur premier ministre. Morin plaçait le français et l'anglais sur un pied d'égalité.

Dans un pays comme celui-ci, ou deux langues sont d'une égale nécessité, les enfants pourront fréquenter une école mixte, surtout pour habituer leurs organes aux sons particuliers de la langue qui leur est la moins familière. ${ }^{29}$

Les Canadiens français, on le voit, ne mettaient pas en doute l'excellence de leur langue maternelle. Leur enthousiasme et la vigueur de leurs prises de position le prouvent largement.

Au cours des premières années de l'Union, cette langue fut d'ailleurs l'objet de nombreuses discussions, réflexions et recherches. Plusieurs nouvelles grammaires furent publiées. Certaines sources anglaises font même état de ces publications. Par deux fois, en septembre 1841 et en juin 1842, le Literay Garland signala la parution de $A$ Treatise on the Pronunciation of the French Language de Jean-Baptiste Meilleur. Cette revue jugeait que l'ouvrage était très intéressant et qu'il aiderait ceux qui désiraient apprendre «la langue élégante dont elle traite ${ }^{30}$. Le magazine estimait qu'il s'agissait là d'une excellente étude sur la langue française «qui dans ce pays est non seulement souhaitable mais nécessaire ${ }^{31}$.

Les Canadiens se préoccupèrent aussi de la qualité de leur langue au Canada. Ainsi, en 1841, Le Canadien salua la publication du

28 La Revue Canadienne, samedi, 4 janvier 1845.

La Minerve, jeudi, 1er janvier 1846.

30 Literary Garland, III, 10 (septembre 1841).

31 Ibid., IV, 7 (juin 1842). 
Manuel des difficultés de la langue française adapté au jeune âge et suivi d'un Recueil de Locutions Vicieuses. L'auteur de ce manuel faisait état des anglicismes qui s'étaient introduits dans le français du Canada ainsi que des néologismes qui le différenciaient du français parlé ailleurs ${ }^{32}$. La publication du Manuel provoqua une tempête dans les encriers. Étienne Parent, rédacteur du Canadien, loua le livre. Bien qu'il ne fut pas totalement d'accord avec son contenu, il était heureux de voir paraître une étude de ce genre car il se rendait compte que la qualité de la langue se détériorait même aux échelons les plus élevés de la société canadienne-française, dans la presse aussi bien qu'au barreau et dans la magistrature. Convaincu que le français était une langue supérieure, Parent s'inquiétait de sa condition au Canada. Dans sa critique du Manuel, il écrivit:

L'auteur fait main basse sur ces locutions barbares, grossières, ridicules, absurdes, qui défigurent la plus polie comme la plus pure des langues modernes. Nous ne parlerons pas des locutions en usage parmi les classes illettrées mais de celles que l'on entend tous ies jours dans ia bonne société. ${ }^{3 \hat{3}}$

Tout simplement, le français était en train de se corrompre. Par exemple, les Canadiens français employaient le mot «office " au lieu de "bureau», des calques de l'anglais telle l'expression "des argents", dérivée du mot anglais "monies", plutôt que "de l'argent ». Que pouvait-on entendre de plus hideux pour une oreille sensible que quelqu'un qui allait acheter des «crackers chez le Groceur ou à la Grossery " ${ }^{34}$ ? Si Parent était d'accord avec l'auteur du Manuel au sujet des erreurs les plus grossières, il ne le suivait plus pour rejeter des mots comme "patate ", "poudrerie " et "traîne" ou autres car il notait que certains de ces mots étaient utilisés en France et que d'autres cadraient parfaitement dans le contexte canadien ${ }^{35}$.

Entre avril et juillet 1842, la Gazette de Québec publia une série d'articles intitulés Questions Grammaticales, qui attaquaient point par point les théories exposées par l'auteur du Manuel. La première escarmouche fut une lettre datée du 13 avril et adressée au rédacteur du Canadien. Le correspondant qui signait $\langle A B C\rangle^{36}$ y faisait remarquer que, dans une description antérieure des exercices

32 Le Canadien, mercredi, 3 novembre 1841.

Ibid., mercredi, 3 novembre 1841 .

Le Canadien, mercredi, 3 novembre 1841 .

Ibid., mercredi, 3 novembre 1841 .

Ibid., samedi, 13 avril 1842 . 
littéraires du couvent des Ursulines de Québec, Parent avait négligé de mentionner la prononciation affectée de la diphtongue «oi». Les jeunes demoiselles du couvent, dans une représentation de la tragédie Esther, avaient prononcé "moi" et "toi» comme "moa" et "toa", plutôt que de respecter la manière habituelle des Canadiens de dire "moé " et "toé". L'article de " $\mathrm{ABC}$ " ne fut pas reçu avec sympathie par le rédacteur du Canadien qui écrivit:

Ces vices de notre prononciation sont si patents que l'on reconnaît partout un Canadien. C'est là nous semble ( sic) une distinction qui n'est pas à envier puisqu'elle dénote une mauvaise éducation ... ${ }^{37}$

Il n'est pas étonnant que les étudiantes du couvent des Ursulines aient été au centre du combat pour une prononciation correcte puisque leur aumônier, l'abbé Thomas Maguire, ancien directeur du Collège de Saint-Hyacinthe, était l'auteur du Manuel ${ }^{38}$. "ABC", l'auteur des articles qui attaquaient Le Manuel, était l'abbé Jérôme Demers, lui-même éducateur et directeur du Séminaire de Québec $^{39}$. Ne se sentant pas particulièrement bien accueilli au Canadien, Demers envoya ses lettres à la Gazette de Québec et Maguire y répondit. Les deux éducateurs échangèrent ainsi toute une correspondance journalistique, dans laquelle ils s'attaquaient à tour de rôle. Ils abordèrent des questions grammaticales; par exemple fallait-il employer l'article ou la préposition devant les noms de ville comme Trois-Rivières et Trois-Pistoles? Fallait-il dire "aux TroisRivières " plutôt que «à Trois-Rivières " et « les Trois-Pistoles " plutôt que "Trois-Pistoles", selon l'usage qui devenait courant ${ }^{40}$ ? Demers prenait particulièrement ombrage de la prononciation de "oi ", affirmant que le "oé" utilisé au Canada était parfaitement acceptable et qu'on l'entendait souvent même dans les rues de $\mathrm{Pa}$ ris ${ }^{41}$. Les néologismes et les canadianismes faisaient évidemment partie de la polémique ${ }^{42}$.

Cette dispute grammaticale ne fut qu'un des nombreux exemples de l'intérêt des Canadiens français pour leur langue au lendemain de l'Union. Une nouvelle grammaire, l'Essai de Grammaire

37 Ibid., samedi, 13 avril 1842.

38 N.E. Dionne, Une dispute grammaticale en 1842: le G.V. Demers vs le G.V. Maguire (Québec, Laflamme et Proulx, 1912), 92. Galerie Historique VII.

39 Ibid., 11.

40 Gazette de Québec, samedi, 21 mai 1842.

41 N.E. Dionne, op. cit., 8.

42 N.E. Dionne, op. cit., 9. 
française suivant les principes de l'Abbé Girard, parut en 1842. Elle était l'oeuvre d'Amable Berthelot. Dans un compte rendu du livre publié dans Le Canadien, François-Xavier Garneau loua l'énergie de l'auteur, mais jugea l'ouvrage un peu trop compliqué et certainement trop avancé pour être employé dans les écoles primaires ${ }^{43}$.

La cause du français au Canada fut certainement favorisée par la nomination du docteur Jean-Baptiste Meilleur au poste de surintendant de l'Instruction Publique pour le Canada-Est en $1842^{44}$. Ce médecin, qui avait été professeur de français au Collège Dartmouth au New Hampshire, était l'auteur d'un manuel de chimie, d'une grammaire anglaise pour les enfants francophones et d'un traité sur la prononciation du français ${ }^{45}$. L'année suivante, en 1843, parut $A$ Theoretical and Practical Grammar de Monsieur de Lévignac. Le Canadien nota que ce livre devrait être bien accueilli par les Anglais de Québec qui désiraient certainement apprendre le français ${ }^{46}$.

Que peut-on conclure de ces quelques réflexions sur la langue française à l'énoṇue de l'Union? D'abord que les Canadiens français restaient fiers de leur langue; puis que les politiciens la défendaient ${ }^{47}$, que des éducateurs ont publié plusieurs livres de grammaire et qu'on trouvait le temps de discuter de la langue jusque dans les moindres détails. On vit même des manuels destinés à l'enseignement du français aux Anglais.

La nouvelle situation créée par l'Union des deux Canadas exigeait plus de vigilance dans la protection du français au Canada. Les mots puissants et éloquents d'Étienne-Pascal Taché, alors député de l'Islet à l'Assemblée législative, montraient bien le danger que couraient les Canadiens français pendant ces années. Pour Taché, c'était tout simplement une question de vie ou de mort pour la nation canadienne.

Bordés comme nous le sommes par les États-Unis, contenant une population instruite, submergés par une émigration égale-

43 Le Canadien, vendredi, 3 juin 1842.

44 Gazette de Québec, mardi, 17 mai 1842.

45 Ibid., samedi, 21 mai 1842.

46 Le Canadien, mercredi, 7 juin 1843.

47 Par exemple, en 1841, pendant la première session du Parlement de l'Union, Étienne Parent rédacteur du Canadien et député de Saguenay, patronna un projet de loi assurant la traduction en français de tous les textes légaux (Québec Gazette, mercredi, ler septembre 1841). Voir aussi: Statutes of Canada (4 et 5 Victoria, cap. 51.) En septembre 1842, Louis-Hippolyte LaFontaine fit son premier discours exclusivement en français, (Debates of the Legislative Assembly, Tuesday, September 13, 1842). 
ment instruite, que nous reste-t-il à faire? Nous instruire comme eux, en intelligence et industrie, et par là conserver notre indépendance comme individus, et notre dignité comme peuple; ou bien, nous familiariser à l'idée affreuse et déchirante de voir nos descendants jouer dans la société le rôle servile et dégradant de manoeuvres, de valets, et de charroyeurs d'eau. ${ }^{48}$

D'autres, comme François-Xavier Garneau, John Neilson, Napoléon Aubin et Étienne Parent, se rendaient également compte de la menace que représentait la nouvelle situation politique.

En fait, cette menace qui pesait sur le français au Canada pendant les années 1840 était sérieuse. Ceux qui définissaient la politique linguistique pour la colonie, soit à Londres, soit au Canada, voulaient la disparition du français. On était conscient de cet objectif dans le Haut et le Bas-Canada. Mais les Canadiens français ont refusé d'accepter l'assimilation. Chose étrange, pour préserver leur culture, ils étaient prêts à apprendre l'anglais. Pourtant, cette réaction hautement pragmatique et réaliste n'indique pas qu'ils désiraient abandonner leur propre langue. La vigueur de leurs attitudes et de leurs sentiments à l'égard du français démontre le contraire. Leurs réactions laissaient prévoir que le français n'allait pas disparaître au Canada. 